\title{
Initial boundary value problems for second order parabolic systems in cylinders with polyhedral base
}

Vu Trong Luong ${ }^{1 *}$ and Do Van Loi ${ }^{2}$

* Correspondence: luongvt2003@yahoo.com

'Department of Mathematics, Taybac University, Sonla city, Sonla, Vietnam

Full list of author information is available at the end of the article

\section{Abstract}

The purpose of this article is to establish the well posedness and the regularity of the solution of the initial boundary value problem with Dirichlet boundary conditions for second-order parabolic systems in cylinders with polyhedral base.

\section{Introduction}

Boundary value problems for partial differential equations and systems in nonsmooth domains have been attracted attentions of many mathematicians for more than last 50 years. We are concerned with initial boundary value problems (IBVP) for parabolic equations and systems in nonsmooth domains. These problems in cylinders with bases containing conical points have been investigated in $[1,2]$ in which the regularity and the asymptotic behaviour near conical points of the solutions are established. Parabolic equations with discontinuous coefficients in Lipschitz domains have also been studied (see [3] and references therein).

In this study, we consider IBVP with Dirichlet boundary conditions for second-order parabolic systems in both cases of finite cylinders and infinite cylinders whose bases are polyhedral domains. Firstly, we prove the well posedness of this problem by Galerkin's approximating method. Next, by this method we obtain the regularity in time of the solution. Finally, we apply the results for elliptic boundary value problems in polyhedral domains given in $[4,5]$ and former our results to deal with the global regularity of the solution.

Let $\Omega$ be an open polyhedral domain in $\mathbb{R}^{n}(n=2,3)$, and $0<T \leq \infty$. Set $Q_{T}=\Omega \times$ $(0, T), S_{T}=\partial \Omega \times(0, T)$. For a vector-valued function $u=\left(u_{1}, u_{2}, \ldots, u_{s}\right)$ and $p=\left(p_{1}\right.$, $\left.p_{2}, \ldots, p_{n}\right) \in \mathbb{N}^{n}$ we use the notation $D^{p} u=\left(\partial_{x}^{p} u_{1}, \ldots, \partial_{x}^{p} u_{s}\right)$.

Let $m, k$ be non negative integers. We denote by $H^{m}(\Omega), \stackrel{\circ}{H}^{m}(\Omega)$ the usual Sobolev spaces as in [6]. By the notation (., .) we mean the inner product in $L_{2}(\Omega)$.

We denote by $H^{m, k}\left(Q_{T}, \gamma\right)(\gamma \in \mathbb{R})$ the weighed Sobolev space of vector-valued functions $u$ defined in $Q_{T}$ with the norm 


$$
\|u\|_{H^{m, k}\left(Q_{T}, \gamma\right)}=\left(\int_{Q_{T}}\left(\sum_{0 \leq|p| \leq m}\left|D^{p} u\right|^{2}+\sum_{j=1}^{k}\left|u_{t}\right|^{2}\right) e^{-\gamma t} d x d t\right)^{1 / 2}<+\infty .
$$

Let us note that if $T<+\infty$, then $H^{m, k}\left(Q_{T}, \gamma\right) \equiv H^{m, k}\left(Q_{T}\right)$.

The space $\stackrel{\circ}{H}^{m, k}\left(Q_{T}, \gamma\right)$ is the closure in $H^{m, k}\left(Q_{T}, \gamma\right)$ of the set consisting of all vectorvalued functions $u \in C^{\infty}\left(Q_{T}\right)$ which vanish near $S_{T}$.

Let $\partial_{\text {sing }} \Omega$ be the set of all singular points of $\partial \Omega$, namely, the set of vertexes of $\Omega$ for the case $n=2$ and the union of all edges of $\Omega$ for the case $n=3$. Let $\rho(x)$ be the distance from a point $x \in \Omega$ to the set $\partial_{\text {sing }} \Omega$. For $a \in \mathbb{R}$, we denote by $H_{a}^{m}(\Omega)$ the weighed Sobolev space of vector functions $u$ defined on $\Omega$ with the norm

$$
\|u\|_{H_{a}^{m}(\Omega)}=\left(\int_{\Omega} \sum_{0 \leq|p| \leq m} \rho(x)^{2(|p|-a)}\left|D^{p} u\right|^{2} d x\right)^{1 / 2}<+\infty .
$$

It is obvious from the definition that continuous imbeddings $H^{m}(\Omega) \subset H_{0}^{m}(\Omega) \subset H_{a-1}^{m}(\Omega)$ hold for all $a \leq 1$.

The weighed Sobolev spaces $H_{a}^{m, k}\left(Q_{T}, \gamma\right), H_{a}^{m}\left(Q_{T}, \gamma\right)$ are defined as sets of all vectorvalued functions defined in $Q_{T}$ with respect to the norms

$$
\|u\|_{H_{a}^{m, k}\left(Q_{T}, \gamma\right)}=\left(\int_{Q_{T}}\left(\sum_{0 \leq|p| \leq m} \rho(x)^{2(|p|-a)}\left|D^{p} u\right|^{2}+\sum_{j=1}^{k}\left|u_{t}\right|^{2}\right) e^{-\gamma t} d x d t\right)^{1 / 2}<+\infty,
$$

and

$$
\|u\|_{H_{a}^{m}\left(Q_{T}, \gamma\right)}=\left(\int_{Q_{T}}\left(\sum_{0 \leq|p|+k \leq m} \rho(x)^{2(|p|+k-a)}\left|D^{p} u_{t^{k}}\right|^{2}\right) e^{-\gamma t} d x d t\right)^{1 / 2}<+\infty .
$$

Let

$$
L(x, t ; D)=-\sum_{i, j=1}^{n} D_{i}\left(A_{i j}(x, t) D_{j}\right)+\sum_{i=1}^{n} B_{i}(x, t) D_{i}+C(x, t),
$$

be a second-order partial differential operator, where $D_{i}=\partial_{x_{i}}$ and $A_{i j}, B_{i}, C$ are $s \times s$ matrices of bounded functions with complex values from $C^{\infty}\left(\overline{Q_{T}}\right), A_{i j}=A_{j i}^{*}, A_{j i}^{*}$ is the transposed conjugate matrix of $A_{j i}$.

We assume that the operator $L$ is uniformly strong elliptic, that is, there exists a constant $C>0$ such that

$$
\sum_{i, j=1}^{n}\left(A_{i j}(x, t) \eta \bar{\eta}\right) \xi_{i} \xi_{j} \geq C|\xi|^{2}|\eta|^{2}
$$

for all $\xi \in \mathbb{R}^{n}, \eta \in \mathbb{C}^{s}$ and a.e. $(x, t) \in Q_{T}$.

In this article, we study the following problem:

$$
u_{t}+L(x, t ; D) u=f \text { in } Q_{T},
$$




$$
\begin{aligned}
& u=0 \text { on } S_{T}, \\
& \left.u\right|_{t=0}=0 \text { in } \Omega,
\end{aligned}
$$

where $f(x, t)$ is given.

Let us introduce the following bilinear form

$$
B(u, v ; t)=\int_{\Omega}\left(\sum_{i, j=1}^{n}\left(A_{i j}(x, t) D_{j} u \overline{D_{i} v}+\sum_{i=1}^{n} B_{i}(x, t) D_{i} u \bar{v}+C(x, t) u \bar{v}\right) d x\right.
$$

Then the following Green's formula

$$
(L(x, t ; D) u, v)=B(u, v ; t)
$$

is valid for all $u, v \in C_{0}^{\infty}(\Omega)$ and a.e. $t \in[0, T)$.

Definition 1.1. A function $u \in \stackrel{\circ}{H}^{1,1}\left(Q_{T}, \gamma\right)$ is called a generalized solution of problem (2) -(4) if and only if $\left.u\right|_{t=0}=0$ and the equality

$$
\left(u_{t}, v\right)+B(u, v ; t)=(f, v), \text { a.e. } t \in[0, T),
$$

holds for all $v \in \stackrel{\circ}{H}^{1}(\Omega)$.

From (1) it follows that there exist constants $\mu_{0}>0, \lambda_{0} \geq 0$ such that

$$
\operatorname{Re} B(u, u ; t) \geq \mu_{0}\|u\|_{H^{1}(\Omega)}^{2}-\lambda_{0}\|u\|_{L_{2}(\Omega)}^{2}
$$

holds for all $u \in \stackrel{\circ}{H}^{1}(\Omega)$ and $t \in[0, T)$. By substituting $u=v e^{-\lambda_{0} t}$ into (2), we can assume for convenience that $\lambda_{0}$ in (6) is zero. Hence, throughout the present paper we also suppose that $B(.$, ; $t)$ satisfies the following inequality

$$
\operatorname{Re} B(u, u ; t) \geq \mu_{0}\|u\|_{H^{1}(\Omega)}^{2}
$$

for all $u \in \stackrel{\circ}{H}^{1}(\Omega)$ and $t \in[0, T)$.

Now, let us present the main results of this article. Firstly, we give a theorem on well posedness of the problem:

Theorem 1.1. Let $f \in L_{2}\left(Q_{T}, \gamma_{0}\right), \gamma_{0}>0$, and suppose that the coefficients of the operator L satisfy

$$
\sup \left\{\left|A_{i j}\right|,\left|B_{i}\right|,|C|: i, j=1, \ldots, n ;(x, t) \in \bar{Q}_{T}\right\} \leq \mu, \mu=\text { const. }
$$

Then for each $\gamma>\gamma_{0}$, problem (2) -(4) has a unique generalized solution $u$ in the space $\stackrel{\circ}{H}^{1,1}\left(Q_{T}, \gamma\right)$ and the following estimate holds

$$
\|u\|_{H^{1,1}\left(Q_{T}, \gamma\right)}^{2} \leq C \mid\|f\|_{L_{2}\left(Q_{T}, \gamma_{0}\right)}^{2}
$$

where $C$ is a constant independent of $u$ and $f$.

Write $A_{i j t^{k}}=\frac{\partial^{k} A_{i j}}{\partial t^{k}}, B_{i t^{k}}=\frac{\partial^{k} B_{i}}{\partial t^{k}}, C_{t^{k}}=\frac{\partial^{k} C}{\partial t^{k}}$. Next, we give results on the smoothness of the solution:

Theorem 1.2. Let $m \in \mathbb{N}^{*}, \gamma_{0}=\frac{2 \mu n}{\mu_{0}}, \sigma=\gamma-\gamma_{0}, \gamma_{k}=(2 k+1) \gamma_{0}$. Assume that the coefficients of L satisfy

$$
\sup \left\{\left|A_{i j t^{k}}\right|,\left|B_{i t^{k}}\right|,\left|C_{t^{k}}\right|: i, j=1, \ldots, n ;(x, t) \in \bar{Q}_{T}, k \leq m+1\right\} \leq \mu, \mu=\text { const. }
$$




\section{Furthermore,}

$$
f_{t^{k}} \in H^{m}\left(Q_{T}, \gamma_{k}\right), \text { for } k=0,1,2 ; f_{t^{k}}(x, 0)=0, \text { for } k=0, \ldots, m-1 .
$$

Then there exists $\eta>0$ such that $u$ belongs to $H_{a+1}^{2+m}\left(Q_{T}, \gamma_{2+m}+\sigma\right)$ for any $|a|<\eta$, and

$$
\|u\|_{H_{a+1}^{2+m}\left(Q_{T}, \gamma_{2+m}+\sigma\right)}^{2} \leq C \sum_{k=0}^{2}\left\|f_{t^{k}}\right\|_{H^{m}\left(Q_{T}, \gamma_{k}\right)}^{2}
$$

where $C$ is a constant independent of $u$ and $f$.

\section{The proof of Theorem 1.1}

Firstly, we will prove the existence by Galerkin's approximating method. Let $\left\{\omega_{k}(x)\right\}_{k=1}^{\infty}$ be an orthogonal basis of $\stackrel{\circ}{H}^{1}(\Omega)$ which is orthonormal in $L_{2}(\Omega)$. Put

$$
u^{N}(x, t)=\sum_{k=1}^{N} C_{k}^{N}(t) \omega_{k}(x),
$$

where $C_{k}^{N}(t), k=1, \ldots, N$, is the solution of the following ordinary differential system:

$$
\left(u_{t}^{N}, \omega_{k}\right)+B\left(u^{N}, \omega_{k} ; t\right)=\left(f, \omega_{k}\right), \quad k=1, \ldots, N,
$$

with the initial conditions

$$
C_{k}^{N}(0)=0, \quad k=1, \ldots, N .
$$

Let us multiply (10) by $C_{k}^{N}(t)$, then take the sum with respect to $k$ from 1 to $N$ to arrive at

$$
\left(u_{t}^{N}, u^{N}\right)+B\left(u^{N}, u^{N} ; t\right)=\left(f, u^{N}\right), t \in[0, T) .
$$

Now adding this equality to its complex conjugate, we get

$$
\frac{d}{d t}\left(\left\|u^{N}\right\|_{L_{2}(\Omega)}^{2}\right)+2 \operatorname{Re} B\left(u^{N}, u^{N} ; t\right)=2 \operatorname{Re}\left(f, u^{N}\right) .
$$

Utilizing (7), we obtain

$$
\operatorname{Re} B\left(u^{N}, u^{N} ; t\right) \geq \mu_{0}\left\|u^{N}\right\|_{H^{1}(\Omega)}^{2} .
$$

By the Cauchy inequality, for an arbitrary positive number $\varepsilon$, we have

$$
2\left|\left(f, u^{N}\right)\right| \leq 2\|f\|_{L_{2}(\Omega)}\left\|u^{N}\right\|_{L_{2}(\Omega)} \leq C\|f\|_{L_{2}(\Omega)}^{2}+\varepsilon\left\|u^{N}\right\|_{L_{2}(\Omega)}^{2}
$$

where $C=C(\varepsilon)$ is a constant independent of $u^{N}, f$ and $t$. Combining the estimates above, we get from (12) that

$$
\frac{d}{d t}\left(\left\|u^{N}(., t)\right\|_{L_{2}(\Omega)}^{2}\right)+2 \mu_{0}\left\|u^{N}(., t)\right\|_{H^{1}(\Omega)}^{2} \leq C \mid\|f(., t)\|_{L_{2}(\Omega)}^{2}+\varepsilon\left\|u^{N}(., t)\right\|_{L_{2}(\Omega)}^{2}
$$

for a.e. $t \in[0, T)$. Now write

$$
\eta(t):=\left\|u^{N}(., t)\right\|_{L_{2}(\Omega)}^{2} ; \xi(t):=\|f(., t)\|_{L_{2}(\Omega)}^{2}, t \in[0, T) .
$$


Then (13) implies

$$
\eta^{\prime}(t) \leq \varepsilon \cdot \eta(t)+C \xi(t), \quad \text { for a.e. } t \in[0, T) .
$$

Thus the differential form of Gronwall-Belmann's inequality yields the estimate

$$
\eta(t) \leq C e^{\varepsilon t} \int_{0}^{t} \xi(s) d s, t \in[0, T)
$$

We obtain from (14) the following estimate:

$$
\left\|u^{N}(., t)\right\|_{L_{2}(\Omega)}^{2} \leq C e^{\left(\varepsilon+\gamma_{0}\right) t} \int_{0}^{t} e^{-\gamma_{0} S}\|f\|_{L_{2}(\Omega)}^{2} d s \leq C e^{\left(\gamma_{0}+\varepsilon\right) t}|| f \|_{L_{2}\left(Q_{T}, \gamma_{0}\right)}^{2} .
$$

Now multiplying both sides of this inequality by $e^{-\gamma t}, \gamma>\gamma_{0}+\varepsilon$, then integrating them with respect to $t$ from 0 to $T$, we obtain

$$
\left\|u^{N}\right\|_{L_{2}\left(Q_{T}, \gamma\right)}^{2} \leq C \mid\|f\|_{L_{2}\left(Q_{T}, \gamma_{0}\right)}^{2} .
$$

Multiplying both sides of (13) by $e^{-\gamma t}$, then integrating them with respect to $t$ from 0 to $\tau, \tau \in(0, T)$, we obtain

$$
\begin{aligned}
& \int_{0}^{\tau} e^{-\gamma t}\left(\frac{d}{d t}\left\|u^{N}\right\|_{L_{2}(\Omega)}^{2}\right) d t+2 \mu_{0} \int_{0}^{\tau} e^{-\gamma t}\left\|u^{N}\right\|_{H^{1}(\Omega)}^{2} d t \\
& \leq C\left(\|f\|_{L_{2}\left(Q_{T}, \gamma_{0}\right)}^{2}+\left\|u^{N}\right\|_{L_{2}\left(Q_{T}, \gamma\right)}^{2}\right)
\end{aligned}
$$

Notice that

$$
\begin{aligned}
\int_{0}^{\tau} e^{-\gamma t}\left(\frac{d}{d t}\left\|u^{N}\right\|_{L_{2}(\Omega)}^{2}\right) d t & =\int_{0}^{\tau} \frac{d}{d t}\left(e^{-\gamma t}\left\|u^{N}\right\|_{L_{2}(\Omega)}^{2}\right) d t+\gamma \int_{0}^{\tau} e^{-\gamma t}\left\|u^{N}\right\|_{L_{2}(\Omega)}^{2} d t \\
& =e^{-\gamma \tau}\left\|u^{N}(x, \tau)\right\|_{L_{2}(\Omega)}^{2}+\gamma \int_{0}^{\tau} e^{-\gamma t}\left\|u^{N}\right\|_{L_{2}(\Omega)}^{2} d t \geq 0 .
\end{aligned}
$$

We employ the inequalities above to find

$$
2 \mu \int_{0}^{\tau} e^{-\gamma t}|| u^{N}\left\|_{H^{1}(\Omega)}^{2} d t \leq C\right\| f \|_{L_{2}\left(Q_{T}, \gamma_{0}\right)}^{2}, \forall \tau \in(0, T)
$$

Since the right-hand side of (16) is independent of $\tau$, we get

$$
\left\|u^{N}\right\|_{H^{1,0}\left(Q_{T}, \gamma\right)}^{2} \leq C\|f\|_{L_{2}\left(Q_{T}, \gamma_{0}\right)^{\prime}}^{2}
$$

where $C$ is a constant independent of $u, f$ and $N$.

Fix any $v \in \stackrel{\circ}{H}^{1}(\Omega)$, with $\|v\|_{H^{1}(\Omega)}^{2} \leq 1$ and write $v=v^{1}+v^{2}$, where $v^{1} \in \operatorname{span}\left\{\omega_{k}\right\}_{k=1}^{N}$ and $\left(v^{2}, \omega_{k}\right)=0, k=1, \ldots, N,\left(v^{2} \in \operatorname{span}\left\{\omega_{k}\right\}_{k=1}^{N}{ }^{\perp}\right)$. We have $\left\|v^{1}\right\|_{H^{1}(\Omega)} \leq\|v\|_{H^{1}(\Omega)} \leq 1$. Utilizing (10), we get

$$
\left(u_{t}^{N}, v^{1}\right)+B\left(u^{N}, v^{1} ; t\right)=\left(f, v^{1}\right) \text { for a.e. } t \in[0, T) \text {. }
$$


From $u^{N}(x, t)=\sum_{k=1}^{N} C_{k}^{N}(t) \omega_{k}$, we can see that

$$
\left(u_{t}^{N}, v\right)=\left(u_{t}^{N}, v^{1}\right)=\left(f, v^{1}\right)-B\left(u^{N}, v^{1} ; t\right)
$$

Consequently,

$$
\left|\left(u_{t}^{N}, v\right)\right| \leq C\left(\|f\|_{L_{2}(\Omega)}^{2}+\left\|u^{N}\right\|_{H^{1}(\Omega)}^{2}\right) .
$$

Since this inequality holds for all $v \in \stackrel{\circ}{H}^{1}(\Omega),\|v\|_{H^{1}(\Omega)} \leq 1$, the following inequality will be inferred

$$
\left\|u_{t}^{N}\right\|_{L_{2}(\Omega)}^{2} \leq C\left(\|f\|_{L_{2}(\Omega)}^{2}+\left\|u^{N}\right\|_{H^{1}(\Omega)}^{2}\right) .
$$

Multiplying (18) by $e^{-\gamma t}, \gamma>\gamma_{0}+\varepsilon$, then integrating them with respect to $t$ from 0 to $T$, and by using (17), we obtain

$$
\left\|u_{t}^{N}\right\|_{L_{2}\left(Q_{T}, \gamma\right)}^{2} \leq C \mid\|f\|_{L_{2}\left(Q_{T}, \gamma_{0}\right)}^{2} .
$$

Combining (17) and (19), we arrive at

$$
\left\|u^{N}\right\|_{H^{1,1}\left(Q_{T}, \gamma\right)}^{2} \leq C\|f\|_{L_{2}\left(Q_{T}, \gamma_{0}\right)^{\prime}}^{2}
$$

where $C$ is a constant independent of $f$ and $N$.

From the inequality (20), by standard weakly convergent arguments, we can conclude that the sequence $\left\{u^{N}\right\}_{N=1}^{\infty}$ possesses a subsequence weakly converging to a function $u \in \stackrel{\circ}{H}^{1,1}\left(Q_{T}, \gamma\right)$, which is a generalized solution of problem (2) -(4). Moreover, it follows from (20) that estimate (8) holds.

Finally, we will prove the uniqueness of the generalized solution. It suffices to check that problem (2)-(4) has only one generalized solution $u \equiv 0$ if $f \equiv 0$. By setting $v=u$ (., $t$ ) in identity (5) (for $f \equiv 0$ ) and adding it to its complex conjugate, we get

$$
\frac{d}{d t}\left(\|u(., t)\|^{2}\right)+2 \operatorname{Re} B(u, u ; t)=0 .
$$

From (7), we have

$$
\frac{d}{d t}\left(\|u\|_{L_{2}(\Omega)}^{2}\right)+2 \mu_{0}\|u\|_{H^{1}(\Omega)}^{2} \leq 0, \quad \text { for a.e. } t \in[0, T) .
$$

Since $\left.u\right|_{t=0}=0$, it follows from this inequality that $u \equiv 0$ on $Q_{T}$. The proof is complete.

\section{The proof of Theorem 1.2}

Firstly, we establish the results on the smoothness of the solution with respect to time variable of the solution which claims that the smoothness depends on the smoothness of the coefficients and the right-hand side of the systems.

To simplify notation, we write

$$
B_{t^{k}}(u, v ; t)=\int_{\Omega}\left(\sum_{i, j=1}^{n}\left(A_{i j t^{k}}(x, t) D_{j} u \overline{D_{i} v}+\sum_{i=1}^{n} B_{i t^{k}}(x, t) D_{i} u \bar{v}+C_{t^{k}}(x, t) u \bar{v}\right) d x .\right.
$$


Proposition 3.1. Let $h \in \mathbb{N}^{*}$. Assume that there exists a positive constant $\mu$ such that (i) $\sup \left\{\left|A_{i j t^{k}}\right|,\left|B_{i t^{k}}\right|,\left|C_{t^{k}}\right|: i, j=1, \ldots, n ;(x, t) \in \bar{Q}_{T}, k \leq h+1\right\} \leq \mu$, (ii) $f_{t^{k}} \in L_{2}\left(Q_{T}, \gamma_{k}\right), k \leq h ; f_{t^{k}}(x, 0)=0,0 \leq k \leq h-1$.

Then for an arbitrary real number $\gamma$ satisfying $\gamma>\gamma_{0}$, the generalized solution $u \in \stackrel{H}{H}^{m, 1}\left(Q_{T}, \gamma\right)$ of problem (2)-(4) has derivatives with respect to $t$ up to order $h$ with $u_{t^{k}} \in \stackrel{\circ}{H}^{1,1}\left(Q_{T}, \gamma_{k}+\sigma\right), k=0, \ldots, h$, and the estimate

$$
\left\|u_{t^{h}}\right\|_{H^{1,1}\left(Q_{T}, \gamma_{h}+\sigma\right)}^{2} \leq C \sum_{j=0}^{h}\left\|f_{t_{i}}\right\|_{L_{2}\left(Q_{T}, \gamma_{j}\right)}^{2}
$$

holds, where $C$ is a constant independent of $u$ and $f$.

Proof. From the assumptions on the coefficients of operator $L$ and the function $f$, it implies that the solution $\left\{C_{k}^{N}\right\}_{k=1}^{N}$ of problem (10)-(11) has derivatives with respect to $t$ up to order $h+1$. We will prove by induction that

$$
\left\|u_{t^{h}}^{N}(., \tau)\right\|_{H^{1}(\Omega)}^{2} \leq C e^{\left(\gamma_{h}+\frac{\sigma}{2}\right) \tau} \sum_{j=0}^{h}\left\|f_{t j}\right\|_{L_{2}\left(Q_{T}, \gamma_{j}\right)}^{2}
$$

and

$$
\left\|u_{t^{h}}^{N}\right\|_{H^{1,0}\left(Q_{T}, \gamma_{h}+\sigma\right)}^{2} \leq C \sum_{j=0}^{h}\left\|f_{t^{j}}\right\|_{L_{2}\left(Q_{T}, \gamma_{j}\right)}^{2} .
$$

Firstly, we differentiate $h$ times both sides of (10) with respect to $t$ to find the following equality:

$$
\left(u_{t^{h+1}}^{N}, \omega_{k}\right)+\sum_{l=0}^{h}\left(\begin{array}{c}
h \\
l
\end{array}\right) B_{t^{h-l}}\left(u_{t^{l}}^{N}, \omega_{k} ; t\right)=\left(f_{t^{h}}, \omega_{k}\right), k=1, \ldots, N .
$$

From the equalities above together with the initial condition (11) and assumption (ii), we can show by induction on $h$ that

$$
\left.u_{t^{k}}^{N}\right|_{t=0}=0 \quad \text { for } k=0, \ldots, h \text {. }
$$

Equality (24) is multiplied by $\frac{d^{h+1} C_{k}^{N}(t)}{d t^{h+1}}$ and sum $k=1, \ldots, N$, so as to discover

$$
\left(u_{t^{h+1}}^{N}, u_{t^{h+1}}^{N}\right)+\sum_{j=0}^{h}\left(\begin{array}{c}
h \\
j
\end{array}\right) B_{t^{h-j}}\left(u_{t^{j}}^{N}, u_{t^{h+1}}^{N} ; t\right)=\left(f_{t^{h}}, u_{t^{h+1}}^{N}\right) .
$$

Adding this equality to its complex conjugate, we get

$$
2\left\|u_{t^{h+1}}^{N}(., t)\right\|_{L_{2}(\Omega)}^{2}+2 \operatorname{Re} \sum_{j=0}^{h}\left(\begin{array}{c}
h \\
j
\end{array}\right) B_{t^{h-j}}\left(u_{t^{\prime}}^{N}, u_{t^{h+1}}^{N} ; t\right)=2 \operatorname{Re}\left(f_{t^{h}}, u_{t^{h+1}}^{N}\right) .
$$

Next, we show that inequalities (22) and (23) hold for $h=0$. According to (26) (with $h=0$ ), we have

$$
2\left\|u_{t}^{N}(., t)\right\|_{L_{2}(\Omega)}^{2}+2 \operatorname{Re} B\left(u^{N}, u_{t}^{N} ; t\right)=2 \operatorname{Re}\left(f, u_{t}^{N}\right) .
$$


Then the equality is rewritten in the form:

$$
2\left\|u_{t}^{N}(., t)\right\|_{L_{2}(\Omega)}^{2}+\frac{\partial}{\partial t} B\left(u^{N}, u^{N} ; t\right)=B_{t}\left(u^{N}, u^{N} ; t\right)+2 \operatorname{Re}\left(f, u_{t}^{N}\right) .
$$

Integrating both sides of this equality with respect to $t$ from 0 to $\tau, \tau \in(0, T)$, employing Garding inequality (7) and Cauchy inequality, and by simple calculations, we deduce that

$$
\left\|u^{N}(., \tau)\right\|_{H^{1}(\Omega)}^{2} \leq \frac{2 \mu n}{\mu_{0}} \int_{0}^{\tau}\left\|u^{N}(., t)\right\|_{H^{1}(\Omega)}^{2} d t+\int_{0}^{\tau}\|f(., t)\|_{L_{2}(\Omega)}^{2} d t .
$$

Thus Gronwall-Belmann's inequality yields the estimate

$$
\begin{aligned}
\left\|u^{N}(., \tau)\right\|_{H^{1}(\Omega)}^{2} & \leq C e^{\gamma_{0} \tau} \int_{0}^{\tau} e^{-\gamma_{0} t}|| f(., t) \|_{L_{2}(\Omega)}^{2} d t \\
& \leq C e^{\gamma_{0} \tau}\|f\|_{L_{2}\left(Q_{T}, \gamma_{0}\right)}^{2}, \quad \text { for all } \tau \in(0, T),
\end{aligned}
$$

where $\gamma_{0}=\frac{2 \mu n}{\mu_{0}}$. Multiplying both sides of (27) by $e^{\left(-\gamma_{0}-\sigma\right) t}$, then integrating them with respect to $t$ from 0 to $T$, we arrive at

$$
\left\|u^{N}\right\|_{H^{1,0}\left(Q_{T}, \gamma_{0}+\sigma\right)}^{2} \leq C\|f\|_{L_{2}\left(Q_{T}, \gamma_{0}\right)}^{2} .
$$

From inequalities (27) and (28), it is obvious that (22) and (23) hold for $h=0$.

Assume that inequalities (22) and (23) are valid for $k=h-1$, we need to prove that they are true for $k=h$. With regard to equality (26), the second term in left-hand side of (26) is written in the following form:

$$
\begin{aligned}
& 2 \operatorname{Re} \sum_{j=0}^{h}\left(\begin{array}{c}
h \\
j
\end{array}\right) B_{t^{h-j}}\left(u_{t^{j}}^{N}, u_{t^{h+1}}^{N} ; t\right) \\
& =2 \operatorname{Re} B\left(u_{t^{h}}^{N}, u_{t^{h+1}}^{N} ; t\right)+2 \operatorname{Re} \sum_{j=0}^{h-1}\left(\begin{array}{c}
h \\
j
\end{array}\right) B_{t^{h-j}}\left(u_{t^{j}}^{N}, u_{t^{h+1}}^{N} ; t\right) \\
& =\frac{\partial}{\partial t}\left[B\left(u_{t^{h}}^{N}, u_{t^{h}}^{N} ; t\right)\right]-B_{t}\left(u_{t^{h}}^{N}, u_{t^{h^{\prime}}}^{N} ; t\right) \\
& +2 \operatorname{Re} \sum_{j=0}^{h-1}\left(\begin{array}{c}
h \\
j
\end{array}\right)\left[\frac{\partial}{\partial t} B_{t^{h-j}}\left(u_{t^{j}}^{N}, u_{t^{h}}^{N} ; t\right)-B_{t^{h-j}}\left(u_{t^{j+1}}^{N}, u_{t^{h}}^{N} ; t\right)-B_{t^{h-j+1}}\left(u_{t^{j}}^{N}, u_{t^{h}}^{N} ; t\right)\right] .
\end{aligned}
$$

Hence, from (26) we have

$$
\begin{aligned}
& 2\left\|u_{t^{h+1}}\right\|_{L_{2}(\Omega)}^{2}+\frac{\partial}{\partial t}\left[B\left(u_{t^{h}}^{N} u_{t^{h}}^{N}, t\right)\right]-B_{t}\left(u_{t^{h}}^{N} u_{t^{h}}^{N}, t\right) \\
& +2 \operatorname{Re} \sum_{j=0}^{h-1}\left(\begin{array}{c}
h \\
j
\end{array}\right)\left[\frac{\partial}{\partial t} B_{t^{h-j}}\left(u_{t^{j}}^{N}, u_{t^{h}}^{N} ; t\right)-B_{t^{h-j}}\left(u_{t^{j+1}}^{N}, u_{t^{h}}^{N} ; t\right)-B_{t^{h-j+1}}\left(u_{t^{j}}^{N}, u_{t^{h}}^{N} ; t\right)\right]=2 \operatorname{Re}\left(f_{t^{h}, u_{h^{h+1}}^{N}}\right) .
\end{aligned}
$$

Integrating both sides of (29) with respect to $t$ from 0 to $\tau, 0<\tau<T$, and using the integration by parts, we find 


$$
\begin{aligned}
& 2\left\|u_{t^{h+1}}\right\|_{L_{2}\left(Q_{\tau}\right)}^{2}+B\left(u_{t^{h}}^{N}, u_{t^{h}}^{N} ; \tau\right) \\
& =\int_{0}^{\tau} B_{t}\left(u_{t^{h}}^{N}, u_{t^{h}}^{N} ; t\right) d t-2 \operatorname{Re} \sum_{j=0}^{h-1}\left(\begin{array}{c}
h \\
j
\end{array}\right) B_{t^{h-j}}\left(u_{t^{j}}^{N}, u_{t^{h}}^{N} ; \tau\right) \\
& +2 \operatorname{Re} \sum_{j=0}^{h-1}\left(\begin{array}{c}
h \\
j
\end{array}\right) \int_{0}^{\tau} B_{t^{h-j+1}}\left(u_{t^{\prime}}^{N}, u_{t^{h}}^{N} ; t\right)+2 \operatorname{Re} \sum_{j=0}^{h-1}\left(\begin{array}{c}
h \\
j
\end{array}\right) \int_{0}^{\tau} B_{t^{h-j}}\left(u_{t^{j+1}}^{N}, u_{t^{h}}^{N} ; t\right) \\
& +2 \operatorname{Re} \int_{Q_{\tau}} f_{t^{h}} \overline{u_{t^{h+1}}^{N}} d x d t .
\end{aligned}
$$

For convenience, we abbreviate by I, II, III, IV, V the terms from the first to the fifth, respectively, of the right-hand side of (30). By using assumption (i) and the Cauchy inequality, we obtain the following estimates:

$$
\begin{aligned}
& \text { (I) } \leq 2 \mu n \int_{0}^{\tau}\left\|u_{t^{h}}\right\|_{H^{1}(\Omega)}^{2} d t . \\
& (\mathrm{II}) \leq C(\varepsilon) \sum_{j=0}^{h-1}\left\|u_{t^{j}}^{N}\right\|_{H^{1}(\Omega)}^{2}+\varepsilon\left\|u_{t^{h}}^{N}\right\|_{H^{1}(\Omega)}^{2} . \\
& \text { (III) } \leq C(\varepsilon) \sum_{j=0}^{h-1} \int_{0}^{\tau}\left\|u_{t^{j}}^{N}\right\|_{H^{1}(\Omega)}^{2}+\varepsilon \int_{0}^{\tau}\left\|u_{t^{h}}^{N}(x, t)\right\|_{H^{1}(\Omega)}^{2} d t . \\
& \text { (IV) } \leq C(\varepsilon) \sum_{j=1}^{h-1} \int_{0}^{\tau}\left\|u_{t^{j}}^{N}\right\|_{H^{1}(\Omega)}^{2}+\varepsilon \int_{0}^{\tau}\left\|u_{t^{h}}^{N}\right\|_{H^{1}(\Omega)}^{2} d t+4 \mu n h \int_{0}^{\tau}\left\|u_{t^{h}}^{N}\right\|_{H^{1}(\Omega)}^{2} d t . \\
& \text { (V) } \leq C\left(\varepsilon_{1}\right) \int_{Q_{\tau}}\left|f_{t^{h}}\right|^{2} d x d t+\varepsilon_{1} \int_{Q_{\tau}}\left|u_{t^{h+1}}^{N}\right|^{2} d x,\left(\varepsilon_{1}<1\right) .
\end{aligned}
$$

Employing the estimates above, we get from (30) that

$$
\begin{aligned}
B\left(u_{t^{h}}^{N}, u_{t^{h}}^{N} ; \tau\right) & \leq C \int_{Q_{\tau}}\left|f_{t^{h}}\right|^{2} d x d t+C_{1} \sum_{j=0}^{h-1} \int_{0}^{\tau}\left\|u_{t^{\prime}}^{N}\right\|_{H^{1}(\Omega)}^{2} d t+2(2 h+1) \mu n \int_{0}^{\tau}\left\|u_{t^{h^{\prime}}}^{N}\right\|_{H^{1}(\Omega)}^{2} d t \\
& +\varepsilon \int_{0}^{\tau}\left\|u_{t^{h}}^{N}\right\|_{H^{1}(\Omega)}^{2} d t+\varepsilon\left\|u_{t^{h}}^{N}\right\|_{H^{1}(\Omega)}^{2}+C_{2} \sum_{j=0}^{h-1}\left\|u_{t^{N}}^{N}\right\|_{H^{1}(\Omega)}^{2} .
\end{aligned}
$$

By using (7) again, we obtain from (31) the estimate

$$
\begin{aligned}
\left\|u_{t^{n}}^{N}\right\|_{H^{1}(\Omega)}^{2} & \leq C \int_{Q_{\tau}}\left|f_{t^{h}}\right|^{2} d x d t+C_{1} \sum_{j=0}^{h-1} \int_{0}^{\tau}\left\|u_{t^{j}}^{N}\right\|_{H^{1}(\Omega)}^{2} d t+C_{2} \sum_{j=0}^{h-1}\left\|u_{t^{j}}^{N}\right\|_{H^{1}(\Omega)}^{2} \\
& +\frac{2(2 h+1) \mu n+\varepsilon}{\mu_{0}-\varepsilon} \int_{0}^{\tau}\left\|u_{t^{h}}^{N}\right\|_{H^{1}(\Omega)}^{2} d t .
\end{aligned}
$$


From (32) and the induction assumptions, we get

$$
\begin{aligned}
\left\|u_{t^{h}}^{N}\right\|_{H^{1}(\Omega)}^{2} & \leq C \int_{Q_{\tau}}\left|f_{t^{h}}\right|^{2} d x d t+C_{1} \sum_{j=0}^{h-1} e^{\gamma_{j} \tau} \int_{0}^{\tau} e^{-\gamma_{j} \tau}\left\|u_{t^{j}}^{N}\right\|_{H^{1}(\Omega)}^{2} d t \\
& +C_{2} \sum_{j=0}^{h-1} e^{\left(\gamma_{j}+\frac{\sigma}{2}\right) \tau} \sum_{k=0}^{j}\left\|f_{t^{k}}\right\|_{L_{2}\left(Q_{T}, \gamma_{k}\right)}^{2}+\frac{2(2 h+1) \mu n+\varepsilon}{\mu_{0}-\varepsilon} \int_{0}^{\tau}\left\|u_{t^{h}}^{N}\right\|_{H^{1}(\Omega)}^{2} d t \\
& \leq C \int_{Q_{\tau}}\left|f_{t^{h}}\right|^{2} d x d t+C_{1} \sum_{j=0}^{h-1} e^{\gamma_{j} \tau}\left\|f_{t^{j}}\right\|_{L_{2}\left(Q_{T}, \gamma_{j}\right)}^{2} \\
& +C_{2} \sum_{j=0}^{h-1} e^{\left(\gamma_{j}+\frac{\sigma}{2}\right) \tau} \sum_{k=0}^{j}\left\|f_{t^{k}}\right\|_{L_{2}\left(Q_{T}, \gamma_{k}\right)}^{2}+\left(\gamma_{h}+\frac{\sigma}{2}\right) \int_{0}^{\tau}\left\|u_{t^{h}}^{N}\right\|_{H^{1}(\Omega)}^{2} d t
\end{aligned}
$$

where $\varepsilon>0$ is chosen such that

$$
\frac{2(2 h+1) \mu n+\varepsilon}{\mu_{0}-\varepsilon}<\frac{2(2 h+1) \mu n}{\mu_{0}}+\frac{\sigma}{2} .
$$

By the Gronwall-Bellmann inequality, we receive from (33) that

$$
\begin{aligned}
\left\|u_{t^{h}}^{N}(., \tau)\right\|_{H^{1}(\Omega)}^{2} & \leq C\left(\int_{Q_{\tau}}\left|f_{t^{h}}\right|^{2} d x d t+\sum_{j=0}^{h-1} e^{\left(\gamma_{j}+\frac{\sigma}{2}\right)^{\tau}}\left\|f_{t^{j}}\right\|_{L_{2}\left(\Omega_{T}, \gamma_{j}\right)}^{2}\right) \\
& +C e^{\left(\gamma_{h}+\frac{\sigma}{2}\right) \tau} \int_{0}^{\tau} e^{-\left(\gamma_{h}+\frac{\sigma}{2}\right) t}\left(\left\|f_{t^{h}}\right\|_{L_{2}(\Omega)}^{2}+\sum_{j=0}^{h-1} e^{\left(\gamma_{j}+\frac{\sigma}{2}\right) t}\left\|f_{t^{\prime}}\right\|_{L_{2}\left(Q_{T}, \gamma_{j}\right)}^{2}\right) d t \\
& \leq C e^{\left(\gamma_{h}+\frac{\sigma}{2}\right) \tau} \sum_{j=0}^{h}\left\|f_{t i}\right\|_{L_{2}\left(Q_{T}, \gamma_{j}\right)^{\prime}}^{2}
\end{aligned}
$$

$\left(\gamma_{h}>\gamma_{j}\right.$, for $\left.j=0, \ldots, h-1\right)$. Now multiplying both sides of this inequality by $e^{\left(-\gamma_{h}-\sigma\right) \tau}$, then integrating them with respect to $\tau$ from 0 to $T$, we arrive at

$$
\left\|u_{t^{h}}^{N}\right\|_{H^{1,0}\left(Q_{T}, \gamma_{h}+\sigma\right)}^{2} \leq C \sum_{j=0}^{h}\left\|f_{t^{\prime}}\right\|_{L_{2}\left(Q_{T}, \gamma_{j}\right)}^{2} .
$$

It means that the estimates (22) and (23) hold for $k=h$.

By the similar arguments in the proof of Theorem 1.1, we obtain the estimate

$$
\left\|u_{t^{h}}^{N}\right\|_{L_{2}\left(Q_{T}, \gamma_{h}+\sigma\right)}^{2} \leq C \sum_{j=0}^{h}\left\|f_{t j}\right\|_{L_{2}\left(Q_{T}, \gamma_{j}\right)}^{2} .
$$

Then the combination between (34) and (35) produces the following inequality:

$$
\left\|u_{t^{h}}^{N}\right\|_{H^{1,1}\left(Q_{T}, \gamma_{h}+\sigma\right)}^{2} \leq C \sum_{j=0}^{h}\left\|f_{t^{j}}\right\|_{L_{2}\left(Q_{T}, \gamma_{j}\right)}^{2} .
$$

Accordingly, by again standard weakly convergent arguments, we can conclude that the sequence $\left\{u_{t^{k}}^{N}\right\}_{N=1}^{\infty}$ possesses a subsequence weakly converging to a function $u^{(k)} \in \stackrel{\circ}{H}^{1,1}\left(Q_{T}, \gamma_{k}+\sigma\right)$. Moreover, $u^{(k)}$ is the $k^{t h}$ generalized derivative in $t$ of the 
generalized solution $u$ of problem (2)-(4). Estimate (21) follows from (36) by passing the weak convergences.

Next, by changing problem (2) -(4) into the Dirichlet problem for second order elliptic depending on time parameter, we can apply the results for this problem in polyhedral domains (cf. $[4,5])$ and our previous ones to deal with the regularity with respect to both of time and spatial variables of the solution.

Proposition 3.2. Let the assumptions of Theorem 3.1 be satisfied for a given positive integer $h$. Then there exists $\eta>0$ such that $u_{t^{k}}$ belongs to $H_{a+1}^{2,0}\left(Q_{T}, \gamma_{k}+\sigma\right)$ for any $|a|$ $<\eta, k=0, \ldots, h$ and

$$
\sum_{k=0}^{h}\left\|u_{t^{k}}\right\|_{H_{a+1}^{2,0}\left(Q_{T}, \gamma_{k}+\sigma\right)}^{2} \leq C \sum_{k=0}^{h}\left\|f_{t^{k}}\right\|_{L_{2}\left(Q_{T}, \gamma_{k}\right)^{\prime}}^{2}
$$

where $C$ is a constant independent of $u$ and $f$.

Proof. We prove the assertion of the theorem by an induction on $h$. First, we consider the case $h=0$. Equalities (2), (3) can be rewritten in the form:

$$
\begin{aligned}
& L(x, t ; D) u=f_{1}:=f-u_{t} \text { in } Q_{T}, \\
& u=0 \text { on } S_{T} .
\end{aligned}
$$

Since $u$ satisfies

$$
B(u, v ; t)=\left(f_{1}, v\right), \forall v \in \stackrel{\circ}{H}^{1}(\Omega) \text { for a.e. } t \in(0, T),
$$

it is clear that for a.e. $t \in(0, T), u$ is the solution of the Dirichlet problem for system (38) with the right-hand side $f_{1}(., t)=f(., t)-u_{t}(., t) \in L_{2}(\Omega) \subset H_{a-1}^{0}$ for all $a \leq 1$. From Theorem 4.2 in [5] (or Theorem 1.1. in [4]), it implies that there exists $\eta>0$ such that $u(., t) \in H_{a+1}^{2}(\Omega)$ for any $|a| \leq \eta$. Furthermore, we have

$$
\|u(., t)\|_{H_{a+1}^{2}(\Omega)}^{2} \leq C|| f_{1}(., t) \|_{H_{a-1}^{0}(\Omega)}^{2} \leq C\left(\|f(., t)\|_{L_{2}(\Omega)}^{2}+\|u(., t)\|_{L_{2}(\Omega)}^{2}\right),
$$

where $C$ is a constant independent of $u, f$ and $t$. Now multiplying both sides of (40)

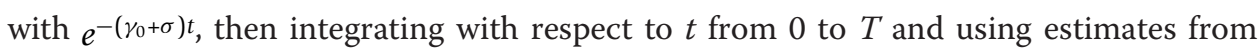
Theorem 3.1, we obtain

$$
\|u\|_{H_{a+1}^{2,1}\left(Q_{T}, \gamma_{0}+\sigma\right)}^{2} \leq C|| f \|_{L_{2}\left(Q_{T}, \gamma_{0}\right)^{\prime}}^{2}
$$

where $C$ is a constant independent of $u, f$. Thus, the theorem is valid for $h=0$. Suppose that the theorem is true for $h-1$; we will prove that this also holds for $h$. By differentiating $h$ times both sides of (38)-(39) with respect to $t$, we get

$$
\begin{aligned}
& L(x, t ; D) u_{t^{h}}=F:=f_{t^{h}}-u_{t^{h+1}}-\sum_{k=0}^{h-1}\left(\begin{array}{l}
h \\
k
\end{array}\right) L_{t^{h-k}}(x, t ; D) u_{t^{k}}, \text { in } Q_{T} \\
& u_{t^{h}}=0, \text { on } S_{T},
\end{aligned}
$$

where

$$
L_{t^{k}}(x, t ; D)=-\sum_{i, j=1}^{n} D_{i}\left(A_{i j t^{k}}(x, t) D_{j}\right)+\sum_{i=1}^{n} B_{i t^{k}}(x, t) D_{i}+C_{t^{k}}(x, t) .
$$


By the induction assumption, it implies that

$$
u_{t^{k}} \in H_{a+1}^{2,1}\left(Q_{T}, \gamma_{k}+\sigma\right) \subset L_{2}\left(Q_{T}, \gamma_{k}+\sigma\right), k=0,1, \ldots, h-1,
$$

and

$$
f_{t^{h}} \in L_{2}\left(Q_{T}, \gamma_{h+1}\right) \subset L_{2}\left(Q_{T}, \gamma_{h}\right) .
$$

Moreover,

$$
u_{t^{h+1}} \in L_{2}\left(Q_{T}, \gamma_{h}\right)
$$

by Theorem 3.1. Hence, for a.e. $t \in(0, T)$, we have $F(., t) \in L_{2}(\Omega) \subset H_{a-1}^{0}(\Omega)$ and the estimate

$$
\|F(., t)\|_{H_{a-1}^{0}(\Omega)}^{2} \leq C\left(\left\|f_{t^{h}(., t)}\right\|_{L_{2}(\Omega)}^{2}+\left\|u_{t^{h+1}}(., t)\right\|_{L_{2}(\Omega)}^{2}+\sum_{k=0}^{h-1}\left\|u_{t^{k}}(., t)\right\|_{L_{2}(\Omega)}^{2}\right)
$$

Applying Theorem 4.2 in [5] again, we conclude from (41)-(42) that $u_{t^{h}}(., t) \in H_{a+1}^{2}(\Omega)$ and

$$
\left\|u_{t^{h}}(., t)\right\|_{H_{a+1}^{2}(\Omega)}^{2} \leq C \| F(., t)_{H_{a-1}^{0}(\Omega)}^{2} .
$$

From the inequality above and (43), it follows that

$$
\left\|u_{t^{h}}(., t)\right\|_{H_{a+1}^{2}(\Omega)}^{2} \leq C\left(\left\|f_{t^{h}(., t)}\right\|_{L_{2}(\Omega)}^{2}+\left\|u_{t^{h+1}(., t)}\right\|_{L_{2}(\Omega)}^{2}+\sum_{k=0}^{h-1}\left\|u_{t^{k}(., t}\right\|_{L_{2}(\Omega)}^{2}\right) .
$$

Multiplying both sides of (44) with $e^{-\left(\gamma_{h}+\sigma\right) t}$, then integrating with respect to $t$ from 0 to $T$ and using Theorem 3.1 with a note that $\gamma_{k}<\gamma_{h}$ for $k=0,1, \ldots, h-1$, we obtain

$$
\left\|u_{t^{h}}\right\|_{H_{a+1}^{2,0}\left(Q_{T,} \gamma_{h}+\sigma\right)}^{2} \leq C \sum_{k=0}^{h}\left\|f_{t^{k}}\right\|_{L_{2}\left(Q_{T}, \gamma_{k}\right)^{\prime}}^{2}
$$

where $C$ is the constant independent of $u$ and $f$. The proof is completed.

Proof of Theorem 1.2. We will prove the theorem by an induction on $m$. It is easy to see that

$$
\|u\|_{H_{a+1}^{2}\left(Q_{T}, \gamma_{2}+\sigma\right)}^{2} \leq \sum_{k=0}^{2}\left\|u_{t^{k}}\right\|_{H_{a+1}^{2-k, 0}\left(Q_{T}, \gamma_{k}+\sigma\right)}^{2}
$$

Hence, Proposition 3.2 implies that the theorem is valid for $m=0$. Assume that the theorem is true for $m-1$, we will prove that it also holds for $m$. It is only needed to show that

$$
\begin{gathered}
u_{t^{s}} \in H_{a+1}^{2+m-s, 0}\left(Q_{T}, \gamma_{2+m-s}+\sigma\right) \quad \text { for } s=m, m-1 \ldots, 0, \text { and } \\
\left\|u_{t^{s}}\right\|_{H_{a+1}^{2+m-s}\left(Q_{T}, \gamma_{2+m-s}+\sigma\right)}^{2} \leq C \sum_{k=0}^{2}\left\|f_{t^{k}}\right\|_{H^{m}\left(Q_{T}, \gamma_{k}\right)}^{2} .
\end{gathered}
$$

Suppose that (45) is true for $s=m, m-1, \ldots, j+1$, return one more to $(41)(\mathrm{h}=\mathrm{j})$, and set $v=u_{t}$, we obtain

$$
L v=F_{j}
$$


where $F_{j}=\left(f_{t j}-v_{t}\right)-(-1)^{m} \sum_{k=1}^{j}\left(\begin{array}{l}j \\ k\end{array}\right) L_{t^{-k}-k} u_{t^{k}}$. By the inductive assumption with respect to $s$, we see that

$$
\begin{gathered}
v_{t} \in H_{a+1}^{1+m-j}(\Omega) \subset H_{a-1}^{m-j}(\Omega) \quad \text { for a.e. } t \in(0, T), \\
f_{t j} \in H^{m}(\Omega) \subset H_{a-1}^{m-j}(\Omega), \quad \text { for a.e. } t \in(0, T),
\end{gathered}
$$

and

$$
L_{t^{-k}-k} u_{t^{k}} \in H^{m-k}(\Omega) \subset H_{a-1}^{m-j}(\Omega), k=1, \ldots, j, \quad \text { for a.e. } t \in(0, T) .
$$

Thus, the right-hand side of (46) belongs to $H_{a-1}^{m-j}(\Omega)$. Applying Theorem 4.2 in [5] again, we get that $v=u_{t^{j}} \in H_{a+1}^{2+m-j}(\Omega)$ for a.e. $t \in(0, T)$. It means that $v=u_{t}$ belongs to $H_{a+1}^{2+m-j, 0}\left(Q_{T}, \gamma_{2+m-j}+\sigma\right)$.

Furthermore, we have

$$
\|v\|_{H_{a+1}^{2+m-j, 0}\left(Q_{T}, \gamma_{2+m-j}+\sigma\right)}^{2} \leq C|| F_{j}\left\|_{H_{a-1}^{h-j, 0}\left(Q_{T}, \gamma_{2+m-j}+\sigma\right)} \leq C \sum_{k=0}^{2}\right\| f_{t^{k}} \|_{H^{m-j}\left(Q_{T}, \gamma_{k}\right)}^{2} .
$$

Therefore,

$$
\begin{aligned}
& \left\|u_{t} \mid\right\|_{H_{1+a}^{2+m-j}}^{2}\left(Q_{T}, \gamma_{2+m-j}+\sigma\right) \\
& \quad \leq\left\|\mid u_{t^{+1}}\right\|_{H_{1+a}^{2+m-j-1}\left(Q_{T}, \gamma_{2+m-j}+\sigma\right)}^{2}+\left\|u_{t}\right\|_{H_{a+1}^{2+m-j, 0}\left(Q_{T}, \gamma_{2+m-j}+\sigma\right)}^{2} \leq C \sum_{k=0}^{2}\left\|f_{t^{k}}\right\|_{H^{m}}^{2}\left(Q_{T}, \gamma_{k}\right) .
\end{aligned}
$$

It implies that (45) holds for $s=j$. The proof is complete for $j=0$.

An example. In order to illustrate the results above, we show an example for the case $L=-\Delta$, and $\Omega$ is a curvilinear polygonal domain in the plane.

Denote by $A_{1}, A_{2}, \ldots, A_{k}$ the vertexes of $\Omega$. Let $\alpha_{j}$ be the opening of the angle at the vertex $A_{j}$. Set

$$
K_{j}=\left\{\left(x_{1}, x_{2}\right) \in \mathbb{R}^{2}: r>0,0<\theta<\alpha_{j}\right\}
$$

as the angle at vertex $A_{j}$ with sides $\gamma_{j}^{-}: \theta=0, \gamma_{j}^{+}: \theta=\alpha_{j}$. Here $r, \theta$ are the polar coordinates of the point $x=\left(x_{1}, x_{2}\right)$, noting that $r(x)=\rho(x)$ is the distance from a point $x \in K_{j} \cap U$ to the set $\left\{A_{1}, A_{2}, \ldots . A_{k}\right\}$, where $U$ is a small neighbourhood of $A_{j}$.

Let $\eta_{j}=\frac{\pi}{\alpha_{j}}$ be the eigenvalue of the pencil $\mathcal{U}(\lambda)$ (cf. [7]) arises from the Dirichlet problem for Laplace operator via the Mellin transformation $r \rightarrow \lambda$. Let $\eta=\min \left\{\eta_{j}\right\}$. We consider the Cauchy-Dirichlet problem for the classical heat equation

$$
\begin{aligned}
& u_{t}-\Delta u=f \text { in } Q_{T}, \\
& u=0 \text { on } S_{T}, \\
& \left.u\right|_{t=0}=0 \text { in } \Omega,
\end{aligned}
$$

where $f: Q_{T} \rightarrow \mathbb{C}$ is given.

Combining Theorem 1.2 and Theorem 4.4 in [5] we receive the following theorem.

Theorem 3.1. Let $\Omega \subset \mathbb{R}^{2}$ be a bounded curvilinear polygonal domain in the plane. 


$$
f_{t^{k}} \in L_{2}\left(Q_{T}, \gamma_{k}\right), \text { for } k=0,1,2 ; f_{t^{k}}(x, 0)=0, \quad \text { for } k=0,1 .
$$

Then the generalized solution $u$ of problem (48)-(50) belongs to $H_{a+1}^{2}\left(Q_{T}, \gamma_{2}+\sigma\right)$ for any $|a|<\eta:=\min \eta_{j}$ as above, and $u$ satisfies the following estimate

$$
\|u\|_{H_{a+1}^{2}\left(Q_{T}, \gamma_{2}+\sigma\right)}^{2} \leq C \sum_{k=0}^{2}\left\|f_{t^{k}}\right\|_{L_{2}\left(Q_{T}, \gamma_{k}\right)}^{2}
$$

where $C$ is a constant independent of $u$ and $f$.

Remark: Let us notice that $f(., t) \in L_{2}(\Omega)=V_{0}^{0}(\Omega), u \in \stackrel{\circ}{H}^{1}(\Omega) \subset V_{0}^{1}(\Omega)$, the weighed Sobolev space $V_{\beta}^{m}(\Omega)$ is defined in [[7], p. 191]. Applying Theorem 6.1.4 in [[7], p. 205] with $l_{2}=2, \beta_{2}=1-a, l_{1}=1, \beta_{1}=0, n=2$ and the strip $0<\operatorname{Re} \lambda<a<\eta$ does not contain any eigenvalue of $\mathcal{U}(\lambda)$, we obtain $u(., t) \in V_{1-a}^{2}(\Omega)$. It is easy to see that $H_{a+1}^{2}(\Omega) \subset V_{1-a}^{2}(\Omega)$. Hence, the regularity of the solution of problem (48)-(50) is better than the regularity result, which can obtain from helps of Theorem 6.1.4 in [[7], p. 205].

\section{Acknowledgements}

This study was supported by the Vietnam's National Foundation for Science and Technology Development (NAFOSTED: 101.01.58.09).

\section{Author details}

'Department of Mathematics, Taybac University, Sonla city, Sonla, Vietnam ${ }^{2}$ Department of Mathematics, Hongduc University, Thanhhoa city, Thanhhoa, Vietnam

\section{Authors' contributions}

All authors typed, read, and approved the final manuscript.

\section{Competing interests}

The authors declare that they have no competing interests.

Received: 18 September 2011 Accepted: 24 December 2011 Published: 24 December 2011

\section{References}

1. Hung, NM, Duong, PT: On the smoothness of generalized solution for parabolic systems in domains with conical points on boundary. Ukrainian Math J. 56(6):854-864 (2004)

2. Hung, NM, Anh, NT: Regularity of solutions of initial-boundary value problems for parabolic equations in domains with conical points. J Diff Equ. 245, 1801-1818 (2008). doi:10.1016/j.jde.2008.07.011

3. Sun-Sig, B: Parabolic equations with BMO coefficients in Lipschitz domains. J Diff Equ. 209, 229-265 (2005). doi:10.1016/ j.jde.2004.08.018

4. Ammann, B, lonescu, A, Nistor, V: Sobolev spaces on Lie manifolds and regularity for polyhedral domains. Documenta Mathematica (electronic). 11, 161-206 (2006)

5. Bacuta, C, Nistor, V, Zikatanov, L: Improving the rate of convergence of "high order finite elements" on polyhedra I: a priori estimates. Numer Func Anal Optim. 26, 613-639 (2005). doi:10.1080/01630560500377295

6. Evans, LC: Partial Differential Equations. Graduate Studies in Mathematics, American Mathematical Society, Providence, RI. 19 (1998)

7. Kozlov, VA, Maz'ya, VG, Rossmann, J: Elliptic Boundary Problems in Domains with Point Singularities. Mathematical Surveys and Monographs, American Mathematical Society, Providence, RI. 52 (1997) 